\title{
Configuration Interactions Constrained by Energy Density Functionals
}

\author{
B. Alex Brown ${ }^{1}$, Angelo Signoracci ${ }^{1}$ and Morten Hjorth-Jensen ${ }^{2}$ \\ ${ }^{1}$ Department of Physics and Astronomy, and National Superconducting Cyclotron Laboratory, \\ Michigan State University, East Lansing, Michigan 48824-1321, USA and \\ ${ }^{2}$ Department of Physics and Center for Mathematical Applications, University of Oslo, N-0316, Oslo, Norway
}

\begin{abstract}
A new method for constructing a Hamiltonian for configuration interaction calculations with constraints to energies of spherical configurations obtained with energy-density-functional (EDF) methods is presented. This results in a unified model that reproduced the EDF binding-energy in the limit of single-Slater determinants, but can also be used for obtaining energy spectra and correlation energies with renormalized nucleon-nucleon interactions. The three-body and/or densitydependent terms that are necessary for good nuclear saturation properties are contained in the EDF. Applications to binding energies and spectra of nuclei in the region above ${ }^{208} \mathrm{~Pb}$ are given.
\end{abstract}

PACS numbers: 26.60.Cs, 21.60.Jz, 27.80.+w

In nuclear structure theory the two main computational methods for heavy nuclei based upon the nucleon fermionic degrees of freedom are the Hartree-Fock or energy-density-functional (EDF) method and the configuration interaction (CI) method. The EDF method is often limited to a configuration with a single Slater determinant. The EDF Hamiltonian has parameters that are fitted to global properties of nuclei such as bindingenergies and rms charge radii [1], [2].

The CI method takes into account many Slater determinants. CI often uses a Hamiltonian derived from experimental single-particle energies and a microscopic nucleon-nucleon interaction [3]. A given CI Hamiltonian is applied to a limited mass region that is related to the configurations of a few valence orbitals outside of a closed shell and the associated renormalized nucleonnucleon interaction that is specific to that mass region [4], 3]. Spectra and binding energies (relative to the closed core) obtained from such calculations for two to four valence particles are in good agreement with experiment [4], 3]. As many valence nucleons are added the agreement with experimental spectra and binding energies deteriorates [5]. An important part that is missing from these CI calculations is the effective two-body interaction that comes from the three-body interaction of two valence nucleons interacting with one nucleon in the core [6]. To improve agreement with experimental spectra one often adjusts some of the valence two-body matrix elements. The most important part of this adjustment can be traced to the monopole component of the two-body matrix elements that controls how the effective single-particle energies evolve as a function of proton and neutron number [6].

Fig. 1 shows Wick's theorem applied to a closed shell for the one-body kinetic energy, the two-body interaction and the three-body interaction. The part contained in the dashed box represents the closed-shell and effective one-body parts of the Hamiltonian that might be contained in an EDF approach. Up to now this has been treated phenomenologically in the framework of the

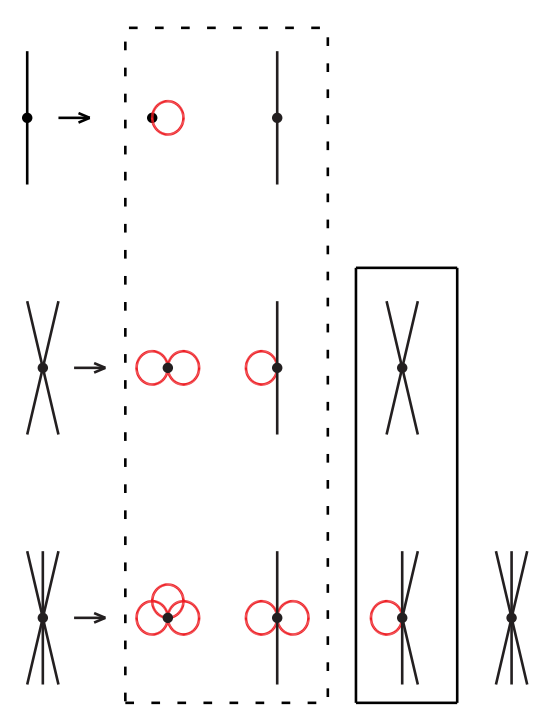

FIG. 1: Schematic diagram for the terms in the Hamiltonian obtained from Wick's theorem for a closed shell. The red lines represent the summation over the orbitals in the closed shell. The black lines represent the valence particles and/or holes.

Skyrme Hartree-Fock or relativistic Hartree method with some parameters (typically 6-10) fitted to global experimental data. There are efforts underway to relate the parameters of these phenomenological approaches to the underlying two and three body forces between nucleons, and also to extend the functional forms to obtain improved agreement with experiment [7]. The part contained in the solid-line box is the residual interaction used for CI calculations. The remaining term is a valence three-body interaction.

In this paper we discuss a new method for obtaining a valence Hamiltonian for valence nucleons outside of a doubly-closed shell. The specific application is made for ${ }^{208} \mathrm{~Pb}$, but it could be applied to any other doubly closedshell system. The single-particle energy for orbital $a$ is 
defined as

$$
e_{a}=E\left({ }^{208} \mathrm{~Pb}+a\right)-E\left({ }^{208} \mathrm{~Pb}\right),
$$

where $E\left({ }^{208} \mathrm{~Pb}\right)$ is the energy of the closed-shell configuration for ${ }^{208} \mathrm{~Pb}$, and $E\left({ }^{208} \mathrm{~Pb}+a\right)$ is the energy of the closed-shell configuration plus one nucleon constrained to be in orbital $a$. Eq. 1 defines the one-body part of the CI calculations. Often experimental data are used for the energies in Eq. 1. In this paper we will use the results of EDF calculations for these energies. The practical use of Eq. 1 requires that two states be connected by a spectroscopic factor of near unity.

The two-body part of the CI Hamiltonian is obtained with the usual renormalization procedure [4]. For our examples, the active valence orbitals are $\left(0 h_{9 / 2}, 1 f_{7 / 2}, 0 i_{13 / 2}\right)$ for protons and $\left(0 i_{11 / 2}, 1 g_{9 / 2}, 0 j_{15 / 2}\right)$ for neutrons. For the two-body valence interaction we use the $\mathrm{N}^{3} \mathrm{LO}$ nucleon-nucleon interaction [8] renormalized to the nuclear medium with the $\mathrm{V}_{\text {lowk }}$ method [9] with a cut-off of $\Lambda=2.2$ $\mathrm{fm}$. Core-polarization corrections are calculated in second-order up to $6 \hbar \omega$ in the excitations energy. We use harmonic-oscillator radial wavefunctions with $\hbar \omega=$ 6.883 MeV.

The new aspect of our method is to take the monopole part of the effective two-body interaction from

$$
\bar{V}_{a b}=E\left({ }^{208} \mathrm{~Pb}+a+b\right)-E\left({ }^{208} \mathrm{~Pb}\right)-e_{a}-e_{b},
$$

where $E\left({ }^{208} \mathrm{~Pb}+a+b\right)$ is the spherical EDF energy of the configuration for a closed shell plus two nucleons constrained to be in orbitals $a$ and $b$. This monopole interaction contains both the two and three body terms shown by the solid-line box in Fig. 1 to the extent that they are contained in the EDF phenomenology. We modify the monopole part of the microscopic valence interaction to reproduce the results of Eq. 2. With this modification, the CI calculations closely reproduce the EDF calculations for single-Slater determinants, even when relatively many valence nucleons are added. Thus, the CI calculations are constrained to reproduce the trends of closed-shell energies and effective single-particle energies obtained with the EDF. For our model space orbitals, Eq. 2 involves about thirty configurations for two nucleons (proton-proton, neutron-neutron and protonneutron), but these calculations in a spherical basis are computationally fast.

For this paper we will use the EDF results based on the Skxm Skyrme interaction [1]. An important property of Skxm is that the experimental single-particle energies for the low-lying single-particle states around ${ }^{208} \mathrm{~Pb}$ are reproduced with an rms deviation of about $300 \mathrm{keV}$. Skxm also has a reasonable value of the incompressibility (234 $\mathrm{MeV})$. We are not aware of any other Skyrme interaction that can do better for the single-particle energies as defined by Eq. 1. For the lowest state for protons $\left(0 h_{9 / 2}\right.$ for $\left.{ }^{209} \mathrm{Bi}\right)$ and neutrons $\left(1 g_{9 / 2}\right.$ for $\left.{ }^{209} \mathrm{~Pb}\right)$, the difference between experiment and theory can be reduced to on the

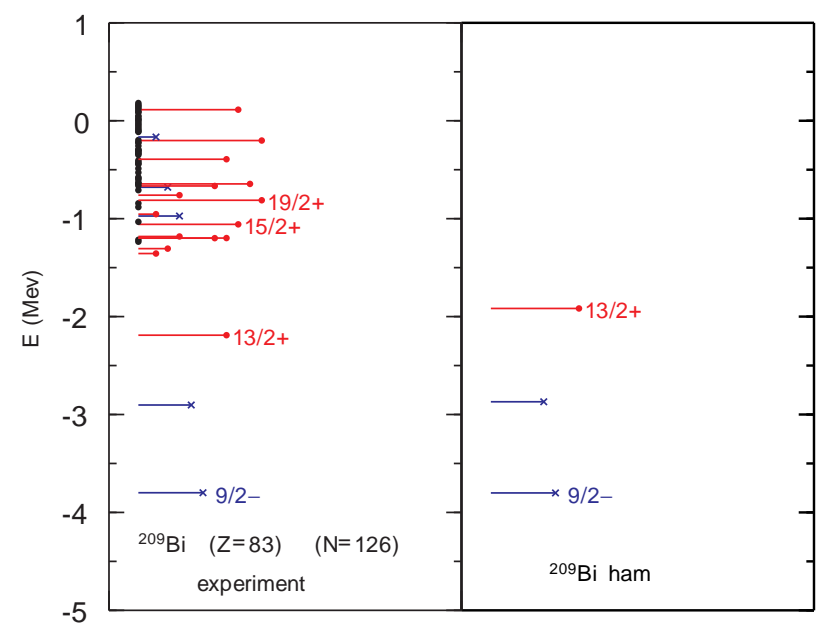

FIG. 2: Comparison of experiment and theory (ham) for ${ }^{209} \mathrm{Bi}$. The energies are with respect to that of ${ }^{208} \mathrm{~Pb}$. The length of the lines indicate the spin with positive parity (red) and negative parity (blue). Experimental levels that are unknown or uncertain are shown by the black dots.

order of $20 \mathrm{keV}$ with only a small increase of $\chi^{2}=0.82$ to $\chi^{2}=0.89$ for all of the data considered in [1]. This is accomplished by using a higher weight for these two data and requires a small adjustment of the Skxm parameters. Since the precise energies of these orbitals are important for the results presented here, we use this new Skyrme interaction called Skxmb. If we use Skxm or any other Skyrme interaction, our conclusions are the same, but the deviation with experiment is worse mainly because the single-particle energies are worse. The binding energy of ${ }^{208} \mathrm{~Pb}$ with Skxmb is $1636.46 \mathrm{MeV}$ compared to the experimental value of $1636.45 \mathrm{MeV}$.

The results obtained from Skxmb for the energies of single-particle states ${ }^{209} \mathrm{Bi}$ relative to the energy of ${ }^{208} \mathrm{~Pb}$ are shown in Fig. 2. The energy of the lowest state, $0 h_{9 / 2}$, is reproduced due to the fit constraint. The next two states (related to the $1 f_{7 / 2}$ and $0 i_{13 / 2}$ orbitals) are also well reproduced. One observes in experiment states related to core-excitation of ${ }^{208} \mathrm{~Pb}$ starting about three $\mathrm{MeV}$ above the ground state.

For the lowest proton orbital with $a=b=\left(0 \mathrm{~h}_{9 / 2}\right)$ the renomalized $\mathrm{N}^{3} \mathrm{LO}$ monopole interaction is $\bar{V}_{\mathrm{N}^{3} \mathrm{LO}}=0.170$ $\mathrm{MeV}$ (it is repulsive due to the Coulomb interaction). The result obtained from Eq. 2 with Skxmb is $\bar{V}_{\text {EDF }}=0.288 \mathrm{MeV}$. The EDF-monopole comes from both terms in the box in Fig. 1 and also contains higher-order contributions implicit in the EDF functional. Whereas, the $\mathrm{N}^{3} \mathrm{LO}$ monopole only contains the valence two-body interaction corrected to second order. The difference is $\bar{V}_{\mathrm{EDF}}-\bar{V}_{\mathrm{N}^{3} \mathrm{LO}}=0.118 \mathrm{MeV}$. This correction is included in CI by modifying all of the valence TBME $<V>_{J}=<$ 


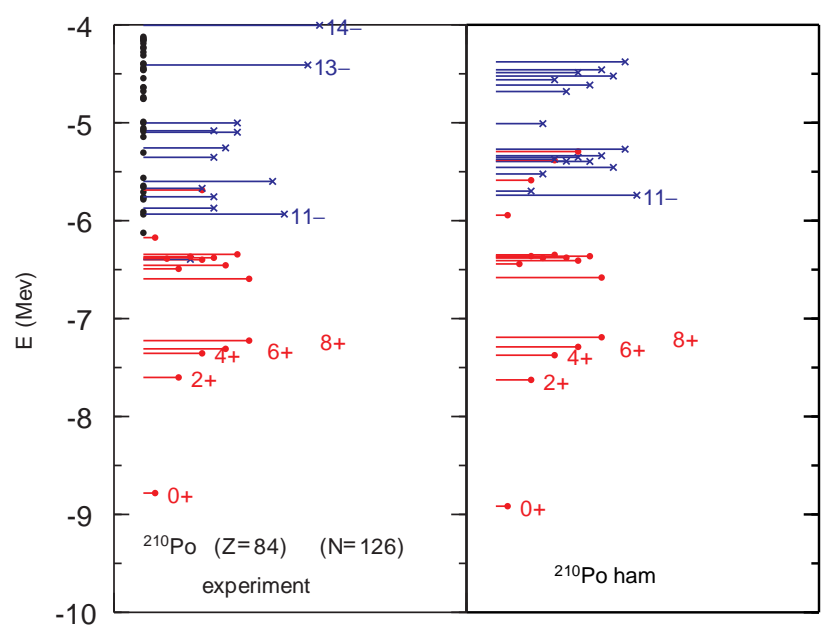

FIG. 3: Comparison of experiment and theory (ham) for ${ }^{210} \mathrm{Po}$ (see caption to Fig. 2).

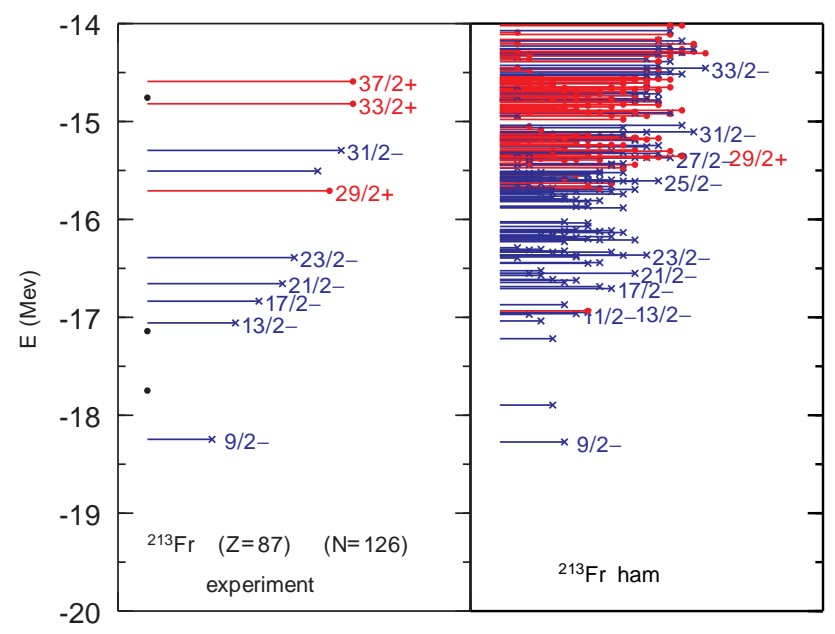

FIG. 4: Comparison of experiment and theory (ham) for ${ }^{213} \mathrm{Fr}$ (see caption to Fig. 2).

$a b J|V| a b J>$ for the $0 h_{9 / 2}$ orbital by

$$
<|V|>_{J, \mathrm{eff}}=<|V|>_{J, \mathrm{~N}^{3} \mathrm{LO}}-\bar{V}_{\mathrm{N}^{3} \mathrm{LO}}+\bar{V}_{\mathrm{EDF}} .
$$

Similar corrections are made for all other diagonal pairs of orbital in the model space.

For the CI calculations we use the code NuShell [10]. The theory Hamiltonian (ham) consists of Skxmb for the single-particle energies, and two-body matrix element obtained from the renormalized $\mathrm{N}^{3} \mathrm{LO}$ interaction corrected to second-order, and then finally the two-body monopoles corrected with Skxmb with Eq. 3. The energies of ${ }^{210} \mathrm{~Pb}$, ${ }^{213} \mathrm{Fr}$ and ${ }^{214} \mathrm{Rn}$ are shown in Fig. 3-5. The agreement

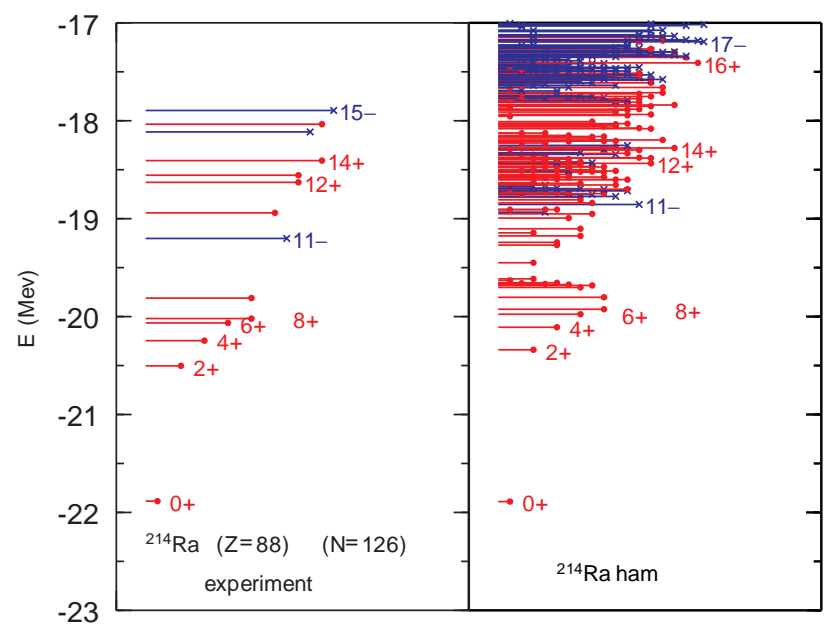

FIG. 5: Comparison of experiment and theory (ham) for ${ }^{214} \mathrm{Ra}$ (see caption to Fig. 2).

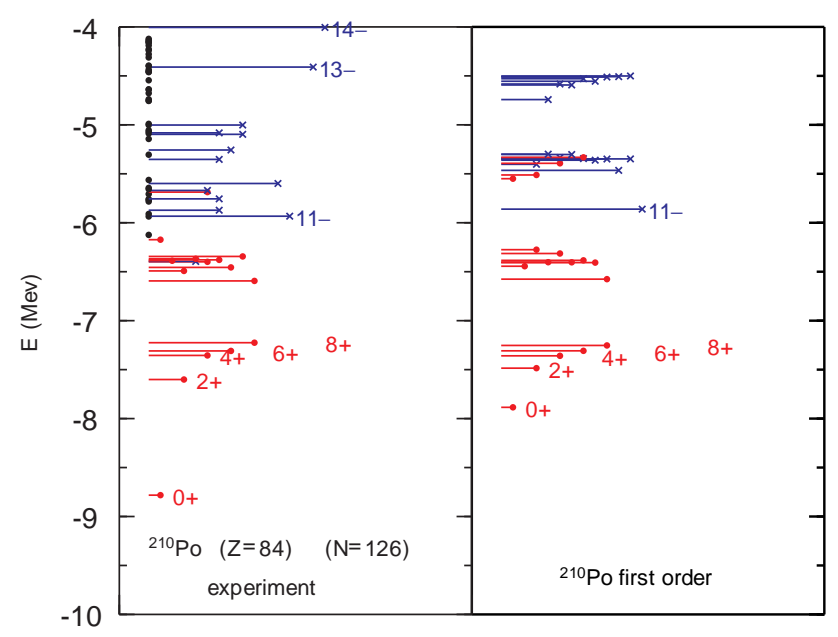

FIG. 6: Comparison of experiment and theory (first order) for ${ }^{210} \mathrm{Po}$ (see caption to Fig. 2).

between experiment and theory is good for the spectra and for the absolute energy relative to ${ }^{208} \mathrm{~Pb}$. For ${ }^{210} \mathrm{Po}$ the agreement between experiment and theory is very good for levels up to three $\mathrm{MeV}$ above the ground state. Above three $\mathrm{MeV}$ the level density of experiment and theory are similar, but one expects additional levels in experiment coming from the core-excitation of ${ }^{208} \mathrm{~Pb}$. For ${ }^{213} \mathrm{Fr}$ and ${ }^{214} \mathrm{Ra}$ the theoretical level density is much higher than experiment because the experimental conditions select mainly the yrast levels. For the low-lying levels in Figs. 3-5 the agreement between the absolute energies of experiment and theory (relative to ${ }^{208} \mathrm{~Pb}$ ) is 


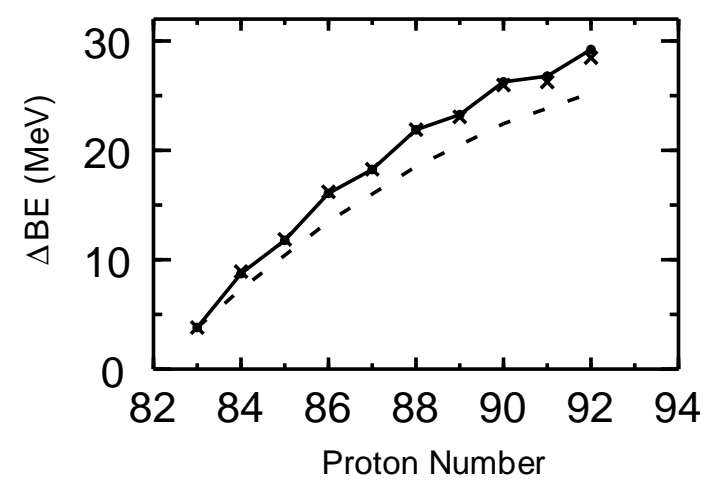

FIG. 7: Binding energies relative to ${ }^{208} \mathrm{~Pb}$. Experiment are the points connected by a line. The results of the $\mathrm{CI}\left(\mathrm{N}^{3} \mathrm{LO}+\mathrm{Skxmb}\right)$ are shown by crosses. The results of the spherical EDF are shown by the dashed line.

usually within $100 \mathrm{keV}$, but there some exceptions with deviations up to about $300 \mathrm{keV}$ (e.g. the $11-$ in ${ }^{214} \mathrm{Ra}$ ). These deviations may be due to many factors such as lack of third-order diagrams, the use of the harmonicoscillator basis for the renormalized $\mathrm{N}^{3} \mathrm{LO}$ matrix elements, non-monopole three-body contributions, or inadequacies in the EDF Skxmb interaction.

When many nucleons are added, the monopole contribution goes as

$$
\Delta E=n(n-1) \bar{V} / 2 \text {, }
$$

where $n$ is the number of valence nucleons. Thus the EDF monopole corrections become much more important as one adds many valence nucleons. When we constrain the CI to the single configuration $\left(0 h_{9 / 2}\right)^{10}$ for the valence protons, the CI calculation gives a binding energy increase of $25.05 \mathrm{MeV}$ (relative to ${ }^{208} \mathrm{~Pb}$ ). The EDF calculation (with the same assumption for the configuration) gives $25.24 \mathrm{MeV}$. These are close to each other due to our EDF monopole correction to the valence matrix elements. If the EDF monopole correction were not included in CI the results would differ by $(45) \mathrm{x}(0.118)$ $=5.3 \mathrm{MeV}$. The microscopic valance interaction on its own is too strong and gives an "over-saturation." The results for the $\left(1 f_{7 / 2}\right)^{8}$ configuration are $13.27 \mathrm{MeV}$ for $\mathrm{CI}$ and and $13.41 \mathrm{MeV}$ for EDF. The difference between CI and EDF might be interpreted in terms of an effective valence three-body monopole interaction with strength $\Delta \mathrm{E}_{3}=25.24-25.05=0.19 \mathrm{MeV}$ for $\left(0 h_{9 / 2}\right)^{10}$ and $\Delta \mathrm{E}_{3}=25.24-25.05=0.14 \mathrm{MeV}\left(1 f_{7 / 2}\right)^{8}$. With $\Delta E_{3}=n(n-1)(n-2) \bar{V}_{3} / 6, \bar{V}_{3}$ is on the order of $1-2$ $\mathrm{keV} . \bar{V}_{3}$ includes the three-body monopole interaction on the right-hand side of Fig. 1, but it may also include other non-quadratic terms that emerge from the EDF solutions. For practical purposes $\Delta \mathrm{E}_{3}$ is small compared to other sources of error in the theory and it may be ignored.

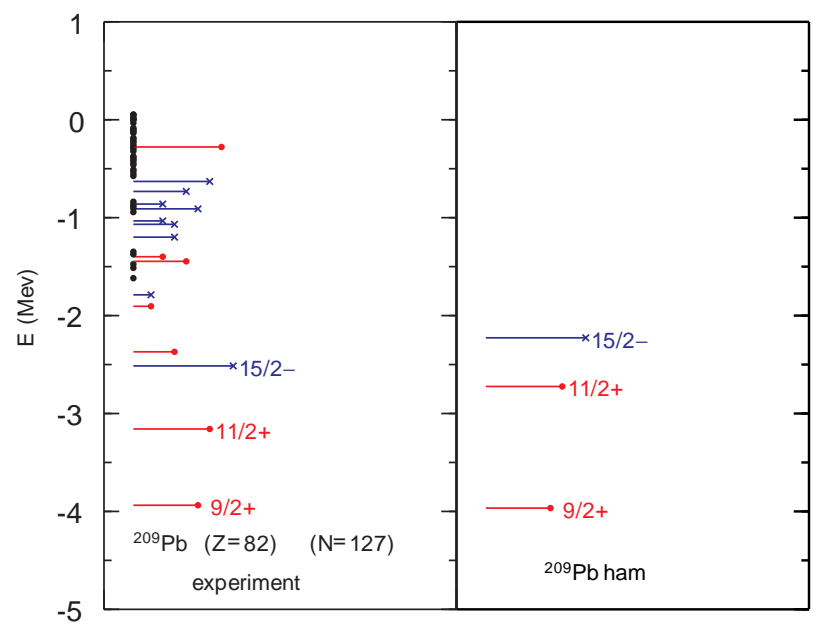

FIG. 8: Comparison of experiment and theory (ham) for ${ }^{209} \mathrm{~Pb}$ (see caption to Fig. 2).

The CI calculation were carried out up to ${ }^{218} \mathrm{U}(Z=$ 92) where the M-scheme dimension is about 1.5 million. The results for the ground state energies are compared to experiment in Fig. 7. The EDF calculation is based upon the spherical $\left(0 h_{9 / 2}\right)^{n}$ configuration with $n=1$ to 10. The difference between EDF and CI can be regarded as the correlation energy in the nuclear ground state, in this case mainly due to the "pairing" interaction. The correlation results in wavefunctions that are highly mixed in the valence proton basis. For example the ground state of ${ }^{218} \mathrm{U}$ contains only $4.7 \%$ of the $\left(0 h_{9 / 2}\right)^{10}$ component. Up to $Z=88$ the difference between experiment and theory for the binding energy relative to ${ }^{208} \mathrm{~Pb}$ is on the order of $100 \mathrm{keV}$, and after this it gradually increases to about $700 \mathrm{keV}$ for ${ }^{218} \mathrm{U}$.

The pairing interaction also appears in Fig. 3 for ${ }^{210} \mathrm{Po}$ by the difference in energy between the ground state and the $\mathrm{J}^{\pi}=8^{+}$state which is dominated $(99.86 \%)$ by the $\left(0 h_{9 / 2}\right)^{2}$ configuration. We show in Fig. 6 the spectrum for ${ }^{210}$ Po obtained from the first-order $\mathrm{N}^{3} \mathrm{LO} \mathrm{V}_{\text {lowk }}$ matrix elements. Comparison with Fig. 3 (which includes second order) shows that two-thirds of the pairing comes from second-order diagrams. The tensor interaction is important for second-order pairing through the bubblediagram which links the valence protons with the core neutrons.

Results for neutrons for the spectra of ${ }^{209} \mathrm{~Pb}$ and ${ }^{210} \mathrm{~Pb}$ are shown in Figs. 8 and 9, respectively. The singleparticle energies of the $0 i_{11 / 2}$ and $0 j_{15 / 2}$ orbitals in ${ }^{209} \mathrm{~Pb}$ are $200-400 \mathrm{keV}$ too high with Skxmb. This is the reason why the theoretical energies of the $10^{+}, 11^{-}$and $13^{-}$ states are too high in ${ }^{210} \mathrm{~Pb}$. The results for ${ }^{210} \mathrm{~Pb}$ are improved when the energies of these two single-particle states are taken from experiment for ${ }^{209} \mathrm{~Pb}$ (left-hand side of Fig. 8) as shown in Fig. 10. For our method to give 


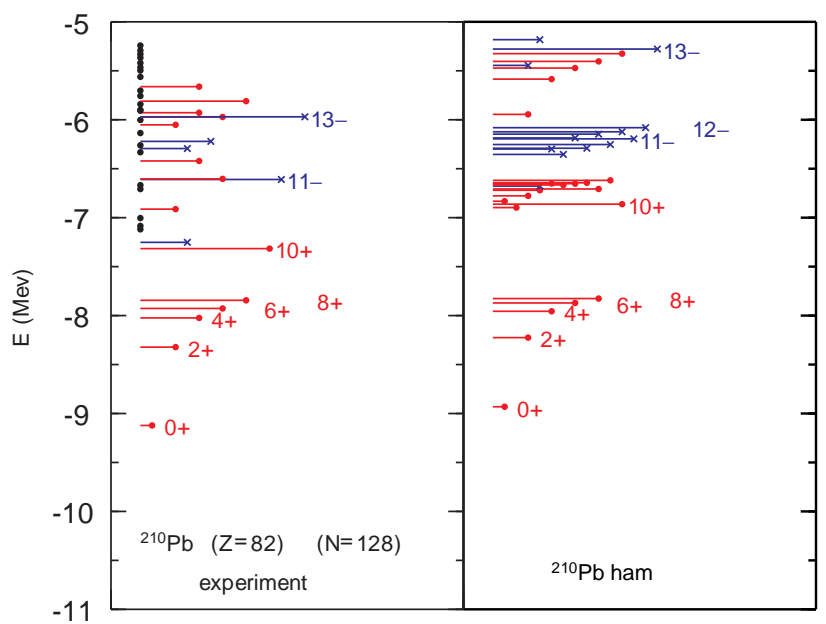

FIG. 9: Comparison of experiment and theory (ham) for ${ }^{210} \mathrm{~Pb}$ (see caption to Fig. 2).

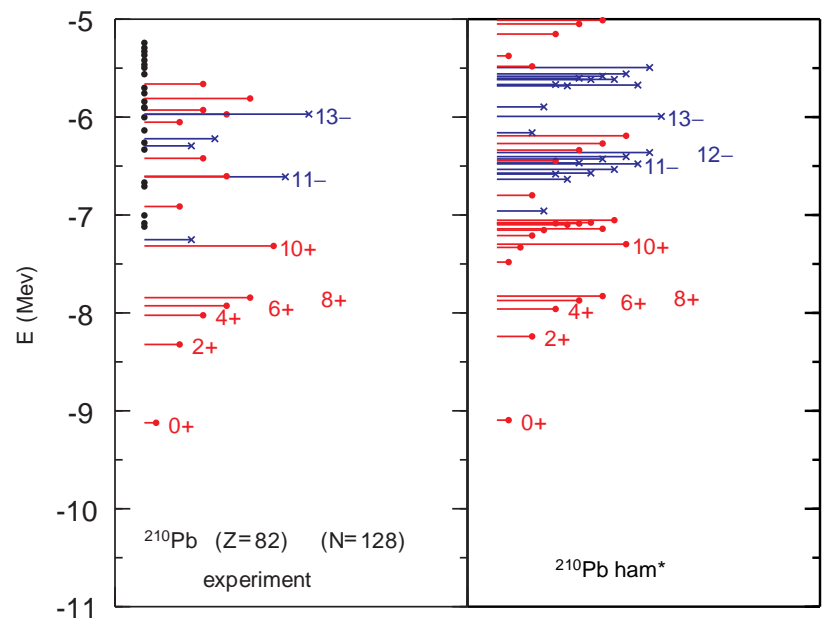

FIG. 10: Comparison of experiment and theory (ham*) for ${ }^{210} \mathrm{~Pb}$ (see caption to Fig. 2). Theory (ham*) is the same as theory (ham) except that the single-particle energies for the neutron $i_{11 / 2}$ and $j_{15 / 2}$ orbitals are taked from the experimental values in ${ }^{209} \mathrm{~Pb}$.

the same results for CI and EDF in the limit of spherical single Slater determinants, one must take both the singleparticle energies and two-particle monopole energies from the EDF calculation; one cannot arbitrarily change the single-particle energies. Thus it is important to obtain EDF functionals that reproduce low-lying single-particle energies near the doubly-magic nuclei.

Results for ${ }^{210} \mathrm{Bi}$ and ${ }^{212} \mathrm{Po}$ are shown in Fig. 11 and 12. Results for the low-lying proton-neutron spectrum of ${ }^{210} \mathrm{Bi}$ are comparable to those shown by [3]. The theo-

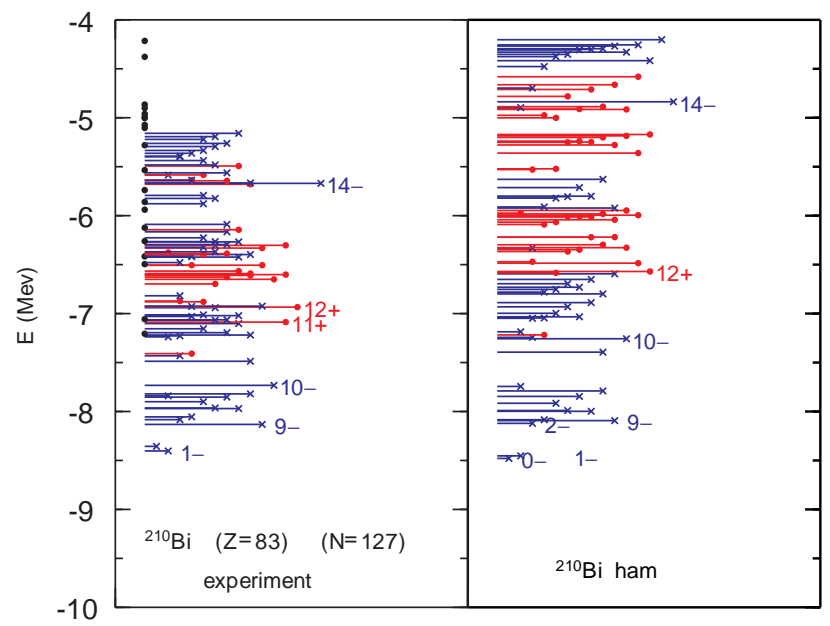

FIG. 11: Comparison of experiment and theory (ham) for ${ }^{210} \mathrm{Bi}$ (see caption to Fig. 2).

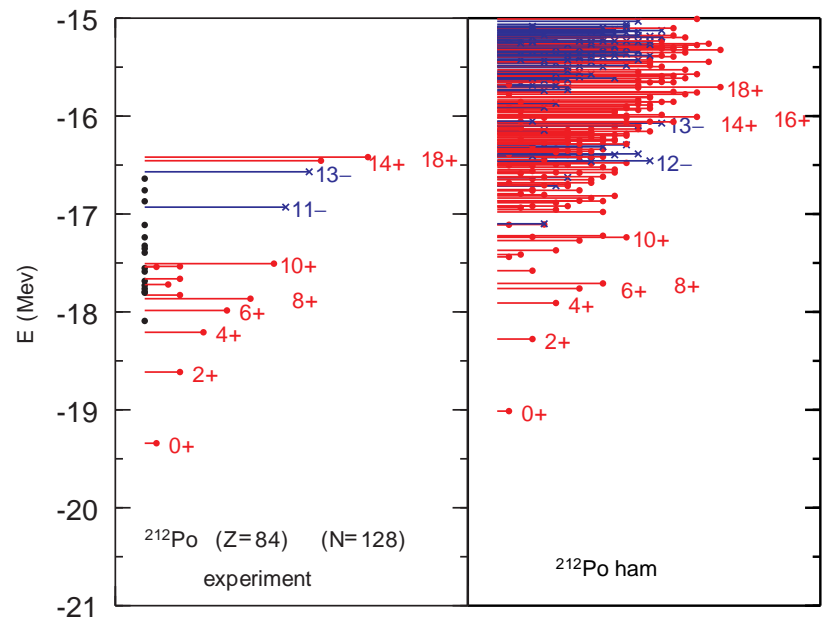

FIG. 12: Comparison of experiment and theory (ham) for ${ }^{212} \mathrm{Po}$ (see caption to Fig. 2).

retical energies for the high-spin state would be in better agreement with experiment if the experimental singleparticle energies from ${ }^{209} \mathrm{~Pb}$ are used for the neutrons. But some specific disagreements remain, for example theoretical the $J^{\pi}=14^{-}$state in ${ }^{210} \mathrm{Bi}$ remains about 500 $\mathrm{keV}$ too high compared to experiment.

The monopole interactions for the $1 g_{9 / 2}$ neutron orbital are $\bar{V}_{\mathrm{N}^{3} \mathrm{LO}}=-0.076 \mathrm{MeV}$ and $\bar{V}_{\mathrm{EDF}}=0.017 \mathrm{MeV}$ giving a correction of $0.017-(0.076)=0.093 \mathrm{MeV}$. The monopole interactions between the $0 h_{9 / 2}$ proton orbital and the $1 g_{9 / 2}$ neutron orbital are $\bar{V}_{\mathrm{N}^{3} \mathrm{LO}}=-0.143 \mathrm{MeV}$ and $\bar{V}_{\text {EDF }}=-0.205 \mathrm{MeV}$ giving a correction of $-0.205-$ 
$(-0.143)=-0.062 \mathrm{MeV}$. Although the EDF monopole corrections are generally positive (leading to less binding), some are negative, as in the last example. This is a result of the microscopic dependence on the specific orbitals being considered and their overlaps with the central proton and neutron densities. The values depend on the isoscalar and isovector properties of the EDF functional that have parameters tuned to reproduce global properties of binding energies.

In conclusion, we have provided a new method that is able to constrain the monopole part of CI calculations to the EDF results in the limit of single-Slater determinants. This constrained CI contains all monopole interactions implicit in EDF including three-body, densitydependent and rearrangement contributions. In the limit of spherical single Slater determinants the CI calculations with this method reproduces the EDF results except for a very small three-body residual. The results for the $N=126$ isotones show that this change in the monopole interaction is crucial for obtaining the correct absolute binding energies. Second-order corrections are important for the pairing interaction. As illustrated in the case of ${ }^{209} \mathrm{~Pb}$ and ${ }^{210} \mathrm{~Pb}$, the accuracy of this method based on
EDF results for the monopole energies plus $\mathrm{N}^{3} \mathrm{LO}$ for the renormalized residual interaction is limited by the accuracy of the EDF methods to reproduce the binding energies for states one nucleon removed from a closed shell (Eq. 1). In our examples for ${ }^{208} \mathrm{~Pb}$ the Skyrme parameters were optimized for the precise ground-state energies of of ${ }^{209} \mathrm{Bi}$ and ${ }^{209} \mathrm{~Pb}$ leaving the rms deviation for all other nuclei about the same as shown in [1]. This method can be applied to any other doubly-closed shell system, but its accuracy will be limited by the accuracy of the EDF results for single-particle energies. Similar local optimizations may be possible for other mass regions. In the coming years we may expect improvements in EDF theory and phenomenology towards a improved universal functional. For cases where the basis dimensions are too large for exact CI methods, it would be interested to apply our Hamiltonian to approximate methods within this model space for valence nucleons outside of ${ }^{208} \mathrm{~Pb}$.

Acknowledgments This work is partly supported by NSF Grant PHY-0758099 and the DOE UNEDF-SciDAC grant DE-FC02-09ER41585.
[1] B. A. Brown, Phys. Rev. C 58, 220 (1998).

[2] P. Klupfel, P. G. Reinhard, T. J. Burvenich and J. A. Maruhn, Phys. Rev. C 79, 034310 (2009).

[3] L. Coraggio, A. Covello, A. Gargano, N. Itaco and T. T. S. Kuo, Prog. in Part. and Nucl. Phys. 62, 135 (2009).

[4] M. Hjorth-Jensen, T. T. S. Kuo and E. Osnes, Phys. Rep. 261, 125 (1995).

[5] B. A. Brown and B. H. Wildenthal, Ann. Rev. of Nucl. Part. Sci. 38, 29 (1988).
[6] A. P. Zuker, Phys. Rev. Lett. 90, 042502 (2003).

[7] J. E. Drut, R. J. Furnstahl and L. Platter, Prog. in Part. and Nucl. Phys. 64, 120 (2010).

[8] D. R. Entem and R. Machleidt, Phys. Rev. C 68, 041001 (2003).

[9] Prog. in Part. and Nucl. Phys. 65, 94 (2010).

[10] Nushell@MSU, B. A. Brown and W. D. M. Rae, MSUNSCL report (2007). 